\title{
Des msw nsw de Thoutmosis III à Deir el-Bahari
}

\author{
Nathalie BeauX
}

\begin{abstract}
Fragments of several identical women holding sistrum and menat necklaces have been found at Deir el-Bahari. These representations were carved on sandstone, erased and recarved after the Amarna period. Proportions and depiction are here very similar to the $m s w n s w$ of Thutmose III at Karnak, where they appear behind the king as he dedicates splendid offerings to Amun-Re on the north wall of the corridor with the Annals. Both scenes are studied and their meaning explored. The fragments, certainly from Thutmose III temple at Deir el-Bahari, may have come from his Hathor shrine.
\end{abstract}

Keywords: Thutmose III, Deir el-Bahari, Karnak, Hathor, $m s w n s w$, royal princesses, sistrum, menat

English title: Msw nsw of Thutmose III at Deir el-Bahari

Nathalie Beaux, chercheur associé de l'Institut français d'archéologie orientale, Le Caire / Collège de France, Paris; nathbeauxgrimal@aol.com

Un lot de quinze fragments de réliefs en grès, représentant des petits personnages féminins, tenant un collier menat et agitant un sistre hathorique, provient de Deir el-Bahari et constitue un ensemble cohérent, objet de cette petite étude. Une reconstitution de la scène est ici proposée, d'après un modèle similaire inscrit sur une paroi du temple d'Amon-Rê de Karnak. La signification des deux représentations est ensuite examinée.

\section{SELECTION DES FRAGMENTS}

Lorsque nous avons entrepris l'étude de la chapelle d'Hathor de Thoutmosis III à Deir el-Bahari, un certain nombre de fragments épars fut identifié, avec l'aide précieuse de la mission du temple de Thoutmosis III, en particulier de Monika Dolińska. Il existait en effet un certain nombre de fragments en grès qui, par leur facture, ne pouvaient appartenir au temple de Mentouhotep, et par leur matière, ne semblaient pas provenir des temples d'Hatchepsout et de Thoutmosis III, dont les bas-reliefs sont principalement en calcaire. 
Le groupe dont il est question dans cet article faisait notamment partie de ces fragments « errants ». Comme la chapelle d'Hathor était essentiellement constituée de blocs en grès, comme l'atteste le sanctuaire actuellement exposé au Musée du Caire ${ }^{1}$, il a été considéré, par défaut, que ces éléments devaient appartenir à ce monument. Un fragment supplémentaire, figurant un sistre, de facture et de taille identiques, se trouve maintenant au Musée de Bolton ${ }^{2}$.

\section{DESCRIPTION DES FRAGMENTS (fig. 1)}

Les fragments en question sont donc tous en grès. Leur décor a été martelé, puis ultérieurement regravé et repeint.

Les personnages figurés, répartis sur au moins deux registres, se succèdent, tournés, à l'exception d'un fragment, vers la gauche. Ces femmes se tiennent debout, un pied légèrement en avant de l'autre, brandissant de la main droite un sistre qu'elles agitent devant elles, tenant un collier menat de l'autre main, qui retombe le long du corps. Elles ont la peau peinte en jaune et une longue perruque noire, dont un pan descend le long du visage jusqu'à la poitrine, l'essentiel de la chevelure rejetée derrière les épaules. Elles sont vêtues d'une longue robe moulante blanche à bretelles, dont émerge un sein nu. Elles portent un collier à cinq rangs, en alternance, vert et bleu, le bas du bijou étant orné de petites perles en forme de gouttes. Elles ont aussi parfois un bracelet au poignet de la main tenant le sistre. Ce dernier présente un manche surmonté d'une tête d'Hathor, couronnée d'un modius, sur lequel est posée la partie musicale de l'instrument, avec trois rangs de sonnailles. Le collier menat comporte encore quelques pastilles bleues dans sa partie arrondie. Aucun nom ou titre ne semble figurer devant les personnages.

\section{COMPARAISON AVEC LES MSW NSW DE KARNAK (fig. 2-3)}

Dans le temple d'Amon-Rê à Karnak, Thoutmosis III consacre lui-même $(\underline{d} s=f)$ une grande offrande à Amon ( $h r p$ mnw), offrande figurée au-dessus du texte des Annales qui se déroule sur l'ensemble du mur nord (dans le couloir nord du sanctuaire de la barque). Derrière cette représentation royale, séparés par une porte, se trouvent six registres de six personnages féminins très semblables à ceux qui nous intéressent : des femmes agitant un sistre et portant de l'autre main un collier menat ${ }^{3}$. La différence est que le bras tenant le collier menat est représenté en avant du corps et non à l'arrière. L'apparence du sistre diffère légèrement : les sonnailles des sistres de Deir el-Bahari sont alignés sur trois rangs, à l'extrémité arrière desquels descend un élément en forme de queue de serpent ${ }^{4}$. En revanche, à Karnak, l'avant de chaque rang de sonnailles prend la forme d'un uraeus dressé,

\footnotetext{
${ }^{1}$ Naville 1907: 36-37.

${ }^{2}$ Ref. $n^{\circ} 1907.90: 17$.

${ }^{3} \mathrm{PM} \mathrm{II}^{2}, 97$ (281)-I : Six rows, women with sistra.

${ }^{4}$ Voir les exemples de sistres avec les rangs de sonnailles figurant un corps entier de serpent, tête dressée à l'avant et queue retombant à l'arrière, donnés par Hickmann 1956: 76-77 et pl. LIII.
} 

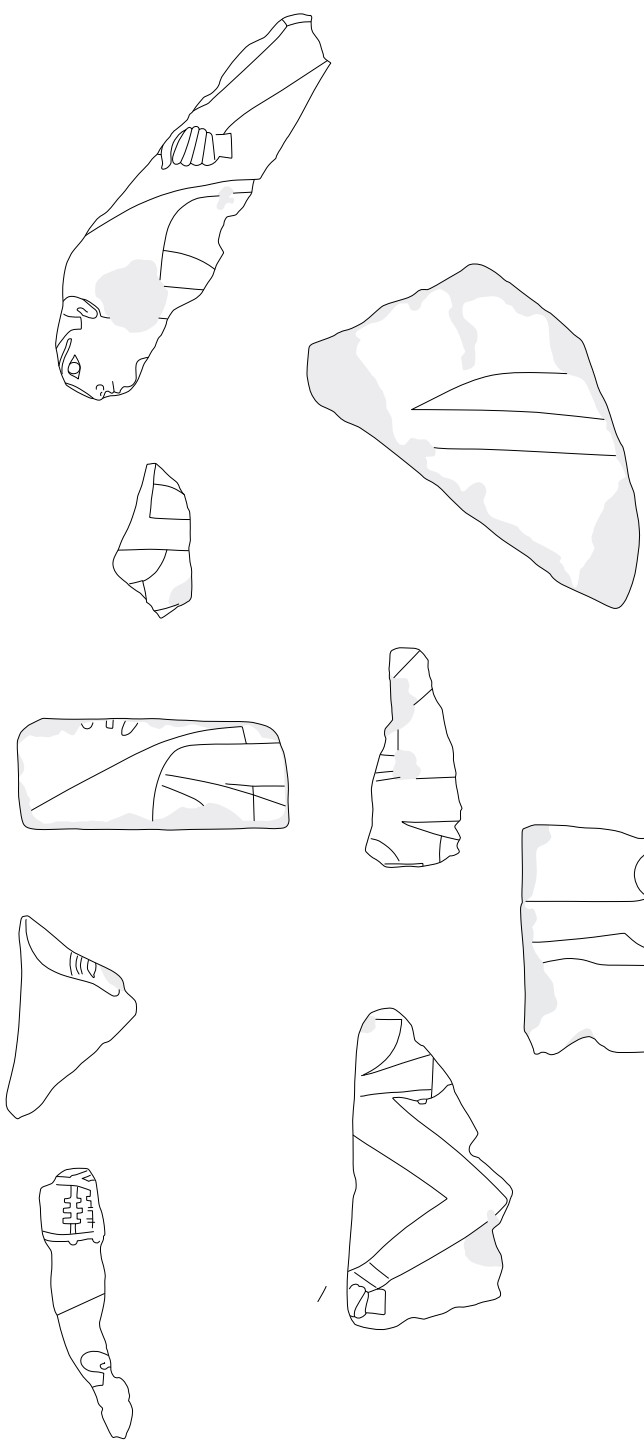
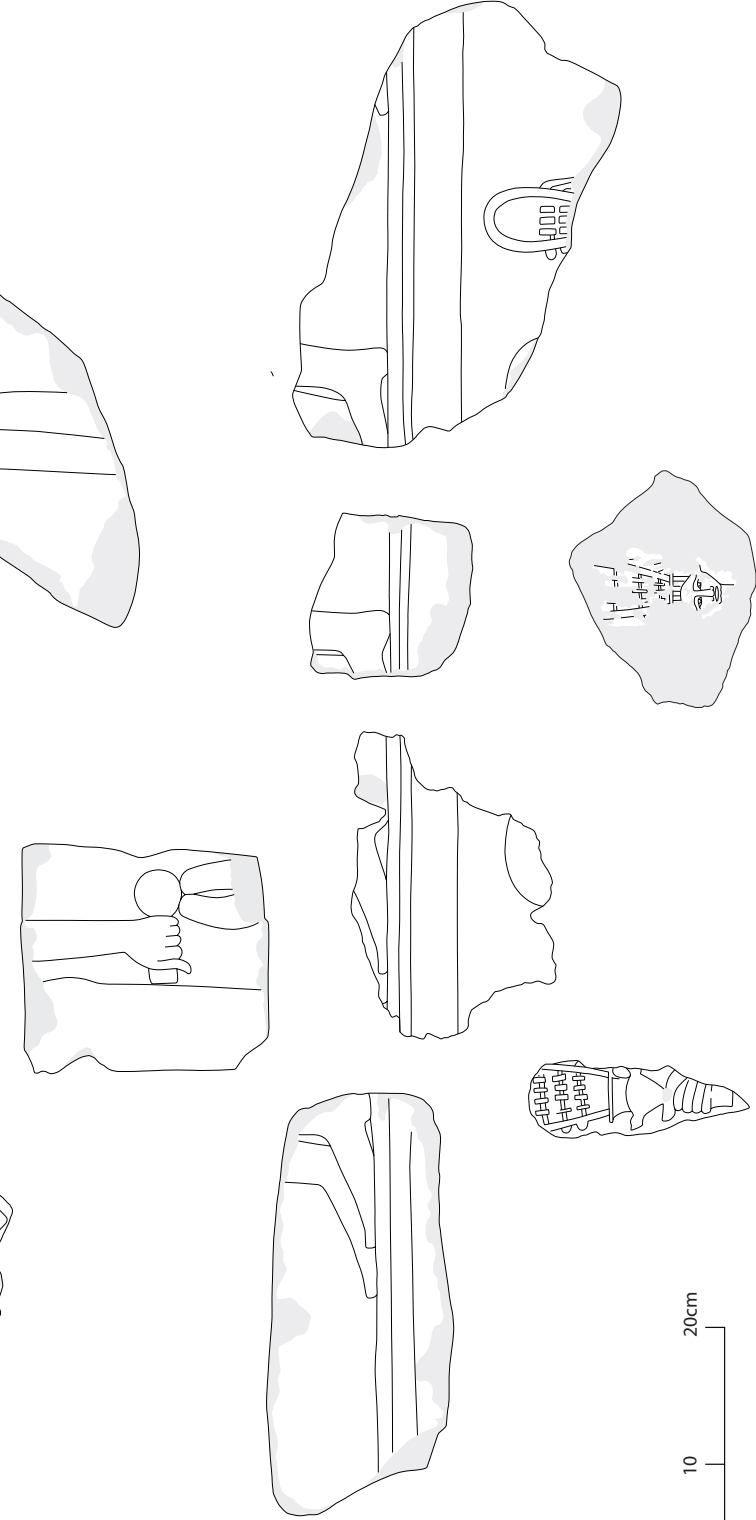

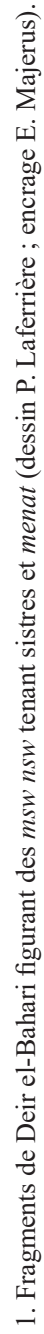




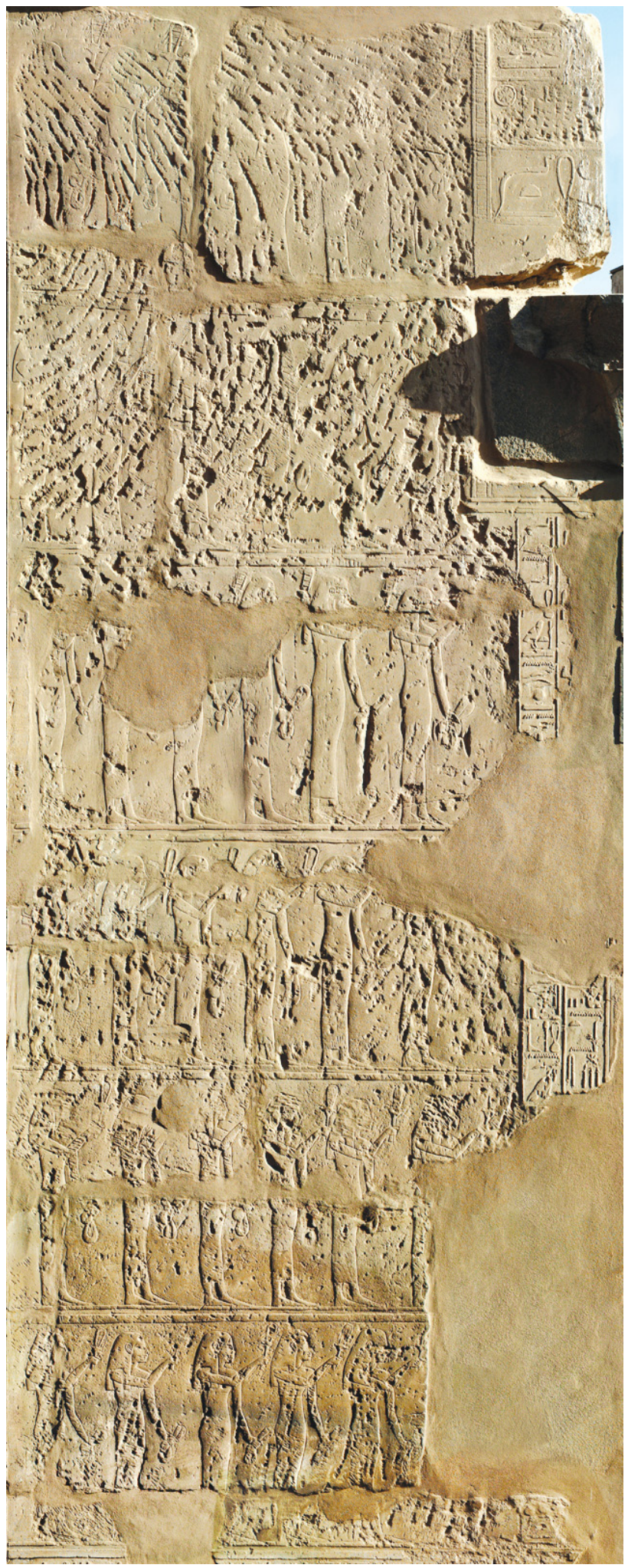

2. Msw nsw représentés derrière Thoutmosis III sur le mur des Annales, dans le temple d'Amon-Rê à Karnak (cliché A. Chéné (C) CNRS-CFEETK). 
3. Détail de la scène représentant $m s w$ nsw derrière Thoutmosis III : mur des Annales, temple d'Amon-Rê, Karnak (cliché N. Beaux).

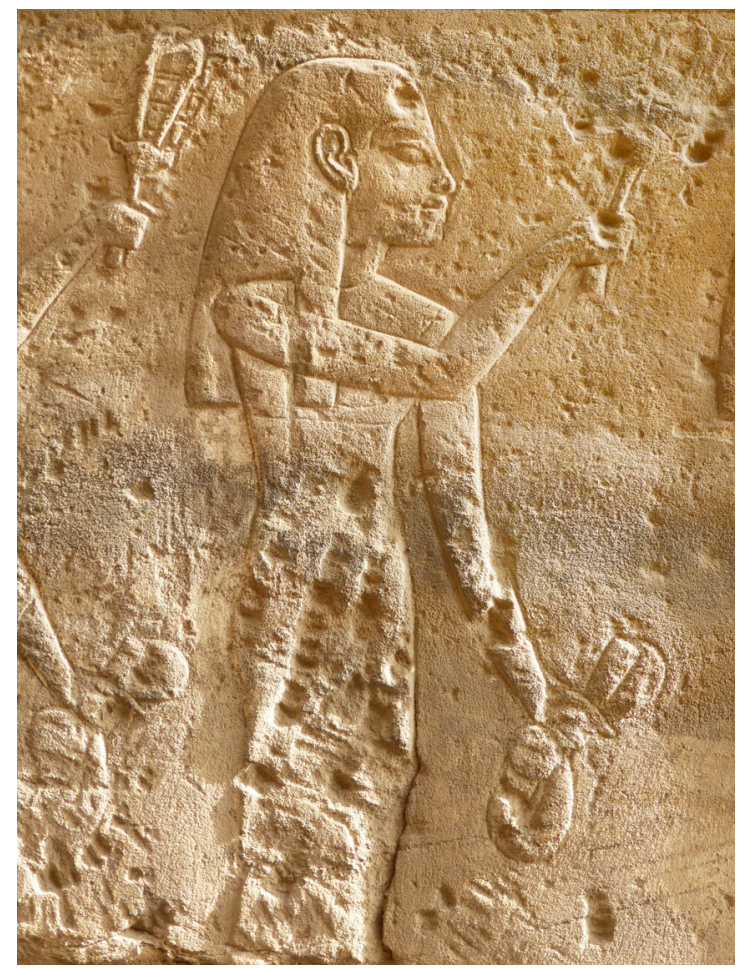

mais l'on n'observe pas d'élément en forme de queue de serpent à l'arrière. La représentation de ces femmes est martelée et regravée, sans qu'aucun nom ou titre ne figure devant elles. Cependant, une double colonne de texte, inscrite devant elles, révèle leur identité : ce sont des $m s w n s w^{5}$.

On note que les dimensions de ces femmes sont identiques à celles de nos personnages à Deir el-Bahari (chaque personnage fait environ $65 \mathrm{~cm}$ de haut). La seule différence est que les $m s w n s w$ de Karnak sont toutes tournées vers la droite alors que celles de Deir el-Bahari vont vers la gauche, à l'exception d'une.

Nous avons donc pris cette scène comme modèle de restitution.

\section{RESTITUTION DE LA SCÈNE (fig. 4)}

Les quinze fragments de Deir el-Bahari permettent de faire un assemblage d'au moins deux registres, chacun de $72 \mathrm{~cm}$ de haut, avec six femmes se dirigeant vers la gauche. Un fragment supplémentaire et de proportion semblable indique qu'un registre, ou une autre scène, figurait au moins un de ces personnages allant dans l'autre direction. Il n'est pas impossible que des inscriptions aient figuré entre les femmes, mais rien ne l'atteste.

\footnotetext{
${ }^{5}$ Urk. IV, 627.
} 
Le contexte dans lequel se situait cette scène est difficile à déterminer, mais il est certain que les petits personnages sont des $m s w n s w$, qui accompagnaient le roi et probablement le suivaient, si l'on compare cette scène avec celle de Karnak. Le panneau sur lequel ils étaient gravés pouvait avoir une certaine ampleur (si on le restaure comme la scène de Karnak, il devait avoir au moins 4,32 m de haut). Il correspond plutôt, s'il appartient bien à la chapelle d'Hathor, à son niveau supérieur ${ }^{6}$.

\section{INTERPRÉTATION}

Georgia Xekalaki a consacré une monographie aux représentations des enfants royaux au Nouvel Empire ${ }^{7}$ et distingue les représentations de princesses dont le nom est donné, de celles qui apparaissent en groupe, de façon le plus souvent anonyme. Elle donne plusieurs exemples de ces groupes de jeunes princesses anonymes, agitant sistre et menat près du roi, à partir d'Amenhotep III ${ }^{8}$. Il faut noter que la scène de Karnak n'a jusqu'ici curieusement été ni identifiée, ni répertoriée, comme une représentation de $m s w n s w^{9}$. Les deux exemples de Karnak et de Deir-el-Bahari seraient donc en fait les premiers du genre dans ce contexte.

En réalité, le thème du groupe de jeunes femmes agitant sistres et menat est connu dès le Moyen Empire. Il est représenté par exemple dans la tombe de Senebi à Meir, où trois jeunes femmes secouent leur sistre et tendent le menat qu'elles portent au cou vers le défunt ${ }^{10}$, un geste légèrement différent de celui qui est présent sur nos fragments, mais qui peut expliquer pourquoi ces instruments se trouvent dans les mains des jeunes femmes. Devant chacune d'elles, une colonne indique que la présentation du collier menat d'Hathor, de ta mère Hathor est opérée pour le ka de Senebi, afin de lui procurer la longévité à laquelle il aspire. Par ailleurs, le Conte de Sinouhé relate la venue devant le roi des enfants royaux, portant sistres et menat et présentant les colliers en invoquant Hathor, invitant le roi à tendre ses mains vers ce qui est bon, (c.-à-d.) les ornements de la Dame du Ciel, afin que l'Or procure la vie à son nez et que la Dame des Étoiles s'unisse à lui (var. le protège) ${ }^{11}$. Le rite de tendre le collier menat au roi est illustré à plusieurs reprises dans les temples, mais c'est une déesse (et pas seulement Hathor) qui accomplit ce geste, la légende accompagnant la représentation faisant écho au texte de Sinouhé ${ }^{12}$.

\footnotetext{
${ }^{6}$ Ce niveau supérieur a été évoqué par Nathalie Beaux (1995: 59-66). Mariusz Caban (voir : la couverture du Polish Archaeology in the Mediterranean 24/2) l'a intégré à sa restitution [3D model of the temples at Deir el-Bahari (West Thebes); state for 2015].

${ }^{7}$ Xekalaki 2011 et particulièrement le Chapitre 4 (« The Representation of King's Daughters in the New Kingdom »).

${ }^{8}$ Xekalaki 2011: 86, 93.

${ }^{9}$ Barguet (1962: 52) les évoque comme « chanteuses d'Amon ».

${ }^{10}$ Blackman 1914: pl. 2-3.

${ }^{11}$ B269-271 (Allen 2015: 141-145).

${ }^{12}$ Desroches-Noblecourt, Kuentz 1968: 181-185. Noter qu'une nouvelle référence de ce type de scène apparaît dans la chapelle d'Hatchepsout à Deir el-Bahari (Beaux et al. 2016: pl. 7a-b). Voir en dernier lieu sur l'offrande du collier menat : Châtelet 2015.
} 


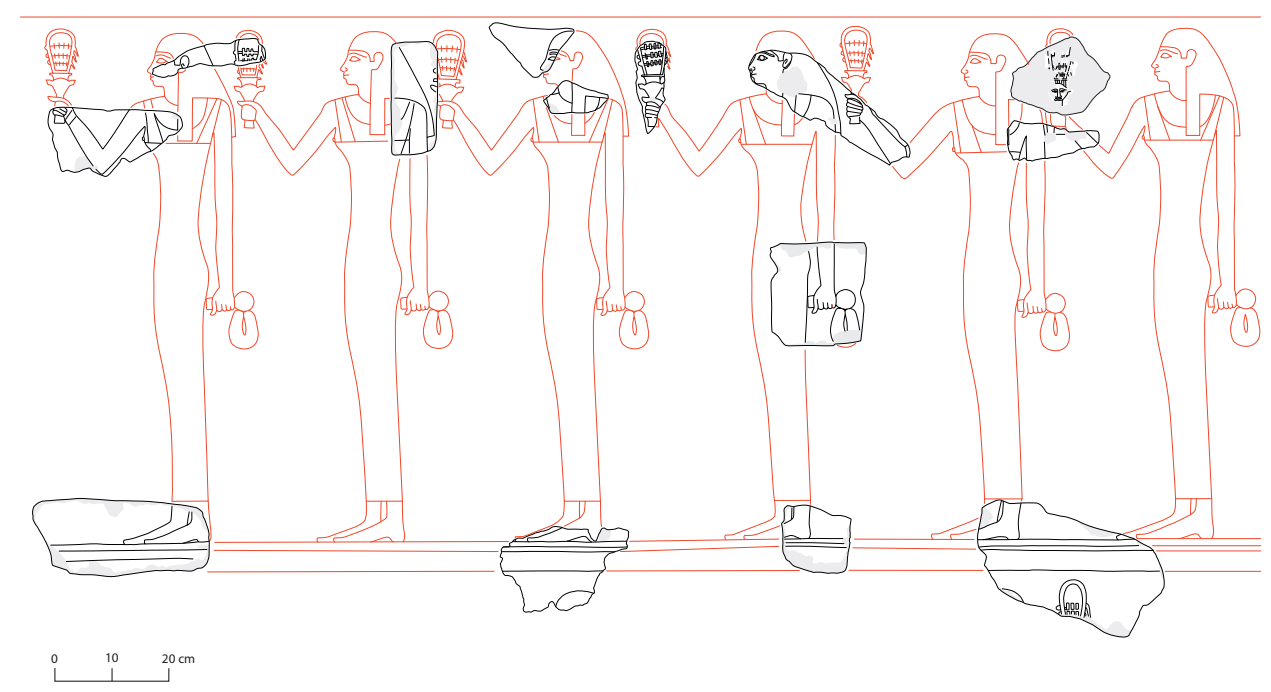

4. Restitution possible de la scène avec les fragments de représentations de $m s w n s w$ trouvés à Deir el-Bahari (dessin P. Laferrière ; encrage E. Majerus).

Le point commun à toutes ces scènes, narrées ou représentées, est l'usage du collier menat et du sistre pour susciter vie, longévité et protection par l'intermédiaire d'une déesse, le plus souvent Hathor. Dans un contexte royal, il s'agit d'évoquer l'union du roi et de la déesse, avec une dimension cosmique ${ }^{13}$. Les princesses, comme les jeunes filles de la tombe de Senebi, par l'entremise du sistre et du menat, mettraient en scène, « réactiveraient » le mythe de cette apparition de la déesse cosmique ${ }^{14}$, «Dame du Ciel, Dame des Étoiles » et des dons qu'elle distribue généreusement. Cependant, il faut être attentif au geste et à la position de ces jeunes filles, dans la scène de Deir el-Bahari comme dans celle de Karnak : le menat et le sistre sont agités vers l'avant à Karnak ; à Deir el-Bahari, le menat est dans la main le long du corps. Les objets symboliques sont là, ils sont activés, mais les princesses (en tout cas pour Karnak) s u i v e n t le roi. Il s'agit donc ici plutôt de référence à la protection, la vie et la longévité acquises auprès de la déesse, après s'être uni à elle, que de mise en scène de l'union proprement dite. Les princesses sont le témoin et l'écho de la relation privilégiée de la déesse avec le roi. À l'époque de Thoutmosis III, point n'est besoin de description de leur chant, ni de donner leur nom, à l'exception de la mention de $m s w n s w{ }^{15}$.

\footnotetext{
${ }^{13}$ «L'hymne récité par les enfants royaux et la scène qu'ils miment devant leur père évoquent l'union d'Atoum et d'Hathor» (Derchain 1970: 79-83).

${ }^{14}$ Daumas 1970: 69-70. Troy (1986: 85-87, 89-91) explique le rôle du sistre lié au mythe de l'œil de Rê, son usage indique le pouvoir de transformation et de renaissance des jeunes princesses, et par conséquent implique le pouvoir de renaissance de leur père, le roi, selon le schéma du Mythe de l'œil de Rê. La princesse/prêtresse joue un rôle vis-à-vis du roi/dieu, l'une incarnant un principe divin féminin (Hathor), l'autre un principe masculin.

${ }^{15}$ Sur les $m s w$ nsw en général, voir : Troy 1986: 89-91.
} 
Leur geste suffit pour évoquer tout l'arrière-plan mythique. La filiation royale ainsi que leur nombre soulignent la puissance vitale du roi autant que sa fertilité. Les princesses ne sont donc pas présentes pour elles-mêmes, mais comme une sorte de miroir, multipliant de façon sonore et visuelle le lien mystique avec la déesse. C'est pourquoi elles conservent non seulement l'anonymat mais aussi une apparence et une gestuelle relativement uniforme et figée. On notera enfin qu'elles sont 36 à Karnak, une allusion possible aux décans, situant alors sur un plan cosmique l'ensemble de la scène, avec le roi comme Horus, fils de Rê, suivi des 36 décans, incarnés par les princesses, devant Amon-Rê, dieu suprême du visible (Rê) et de l'invisible (Amon), dans « l'aura » d'Hathor, « Dame du ciel, Dame des étoiles », dont la présence est annoncée par le concert des sistres et des menat. En effet, ces instruments évoquent mythiquement le bruit du passage de la vache sacrée dans les papyrus ${ }^{16}$, son approche bienfaisante et salvatrice pour le jeune Horus - comme la statue de la chapelle d'Hathor à Deir el-Bahari ${ }^{17}$ le figure : la vache divine fendant les papyrus, protège de son cou le petit roi qui marche devant elle. Elle est donc sur les pas du roi, on l'entend déjà venir grâce aux instruments qui s'agitent derrière lui.

Dans la scène de Karnak comme dans celles, plus tard, de Soleb, les $m s w n s w$ apparaissent lors d'une cérémonie exceptionnelle : à Karnak, c'est l'offrande magnifique à Amon-Rê, fruit des campagnes victorieuses du roi ; à Soleb ${ }^{18}$, c'est la célébration de la fête $S e d$ d'Aménophis III et l'Érection du pilier djed dans la tombe de Kherouef ${ }^{19}$. Il est difficile de dire quel était le contexte de la scène de Deir el-Bahari, mais il était certainement exceptionnel. Son emplacement est lui aussi impossible à déterminer, mais il est probable qu'il demandait une paroi de grande envergure, si l'on y voit une scène aussi importante que celle de Karnak, avec $36 \mathrm{msw} n s w$ sur six registres.

\section{CONCLUSION}

Les fragments des bas-reliefs représentant des femmes tenant sistre et menat retrouvés à Deir el-Bahari peuvent être assemblés en une scène de plusieurs registres, écho d'un tableau similaire figuré derrière Thoutmosis III lors de sa consécration de la grande offrande sur le mur nord des Annales, dans le temple d'Amon-Rê à Karnak. On peut sans doute les replacer dans le niveau supérieur de la chapelle d'Hathor de Thoutmosis III à Deir el-Bahari, mais rien ne le prouve.

Les jeunes filles ne semblent pas nommées, portent toutes le même vêtement et affectent une pose identique, agitant sistre et menat. Elles sont volon t a i re ment a n o n y mes et seulement décrites, à Karnak, comme $m s w n s w$. Leur présence d e r r i è r e le roi (à Karnak, et probablement aussi à Deir el-Bahari) a pour but de l'entourer d'une référence mythique

\footnotetext{
${ }^{16}$ Hickmann 1956: 21. Il évoque les tiges de papyrus qu'on cueillait dans les marais du Nil pour la fête d'Hathor et que l'on secouait rythmiquement (ce qui donna son nom au sistre). Voir aussi Aufrère 1998.

${ }^{17} \mathrm{PM} \mathrm{II}^{2}, 380,381$.

${ }^{18}$ Giorgini, Robichon, Leclant 1998: pl. 94, 127, 130-131.

${ }^{19}$ Epigraphic Survey 1980: pl. 57 ; Wente 1969: 83-91.
} 
sonore, témoignant, par leur filiation et par leur geste, de son lien étroit et constamment réactivé avec la déesse cosmique, « Dame du Ciel, Dame des Étoiles, Or », déesse qui lui accorde souffle de vie et longévité.

Ces multiples $m s w n s w$ anonymes, agitant sistres et menat, auprès de Thoutmosis III, à Karnak et à Deir el-Bahari, sont les premières représentations connues du genre dans ce type de contexte.

\section{Remerciements}

L'étude de la chapelle d'Hathor de Thoutmosis III a débuté sur le terrain en 1994, sur la suggestion du professeur Jadwiga Lipińska, avec l'appui de l'IFAO et l'accord du CSA. La publication du monument est en cours d'élaboration et paraîtra dans les MIFAO. Je suis reconnaissante à Monika Dolińska pour tous les échanges fructueux au cours de cette recherche et notamment pour m'avoir indiqué l'existence du fragment du Musée de Bolton figurant un sistre. Je remercie également Nicolas Grimal, qui prépare la publication du texte des Annales de Thoutmosis III et de la grande scène d'offrande, de m'avoir autorisée à publier cette photographie prise par André Chéné pour le CFEETK.

\section{Références}

Allen, J.P. 2015: Middle Egyptian Literature. Eight Literary Works of the Middle Kingdom, Cambridge

Aufrère, S. 1998: Flore pharaonique et croyances égyptiennes, BSEG 22, 5-16

Barguet, P. 1962: Le temple d'Amon-Rê à Karnak. Essai d'exégèse, RAPH 21, Le Caire

Beaux, N. 1995: La chapelle d'Hathor de Thoutmosis III à Deir-el-Bahari, VarAeg 10/2-3, 59-66

Beaux, N., Karkowski, J., Majerus, E., Pollin, G. 2016: La chapelle d'Hathor. Temple d'Hatchepsout à Deir el-Bahari. II - Façade et salles hypostyles. 1 - Figures et planches, MIFAO 133, Le Caire

Blackman, A.M. 1914: The Rock Tombs of Meir I: The Tomb-Chapel of Ukh-Hotp's Son Senbi, ASEg 22, London

Châtelet, C. 2015: L'offrande du collier-menit dans les temples d'époque gréco-romaine, MRE 16, Bruxelles

Daumas, F. 1970: Les objets sacrés de la déesse Hathor à Dendara, $R d E$ 22, 63-78

Derchain, P. 1970: La réception de Sinouhé à la cour de Sésostris I ${ }^{\mathrm{er}}, R d E$ 22, 79-83

Desroches-Noblecourt, Chr., Kuentz, Ch. 1968: Le petit temple d'Abou Simbel. « Nofretari pour qui se lève le dieu-soleil » I: Étude archéologique et épigraphique. Essai d'interprétation, Le Caire

Epigraphic Survey 1980: The Tomb of Kheruef. Theban Tomb 192, OIP 102, Chicago, Ill. Giorgini, M.S., Robichon, C., Leclant, J. 1998: Soleb V: Le temple. Bas-reliefs et inscriptions, éd. Beaux, N., Le Caire

Hickmann, H. 1956: 45 siècles de musique dans l'Égypte ancienne, à travers la sculpture, la peinture, l'instrument, Paris 
Naville, E. 1907: The XI ${ }^{\text {th }}$ Dynasty Temple at Deir el-Bahari I, MEEF 28, London

Troy, L. 1986: Patterns of Queenship in ancient Egyptian myth and history, Boreas 14, Uppsala

Wente, E.F. 1969: Hathor at the Jubilee, [dans :] Hauser, E.B. (éd.), Studies in honor of John A. Wilson, SAOC 35, Chicago, Ill., 83-91

Xekalaki, G. 2011: Symbolism in the Representation of the Royal Children during the New Kingdom, BAR-IS 2314, Oxford 


\section{ÉTUDES et TRAVAUX XXX / 2017}

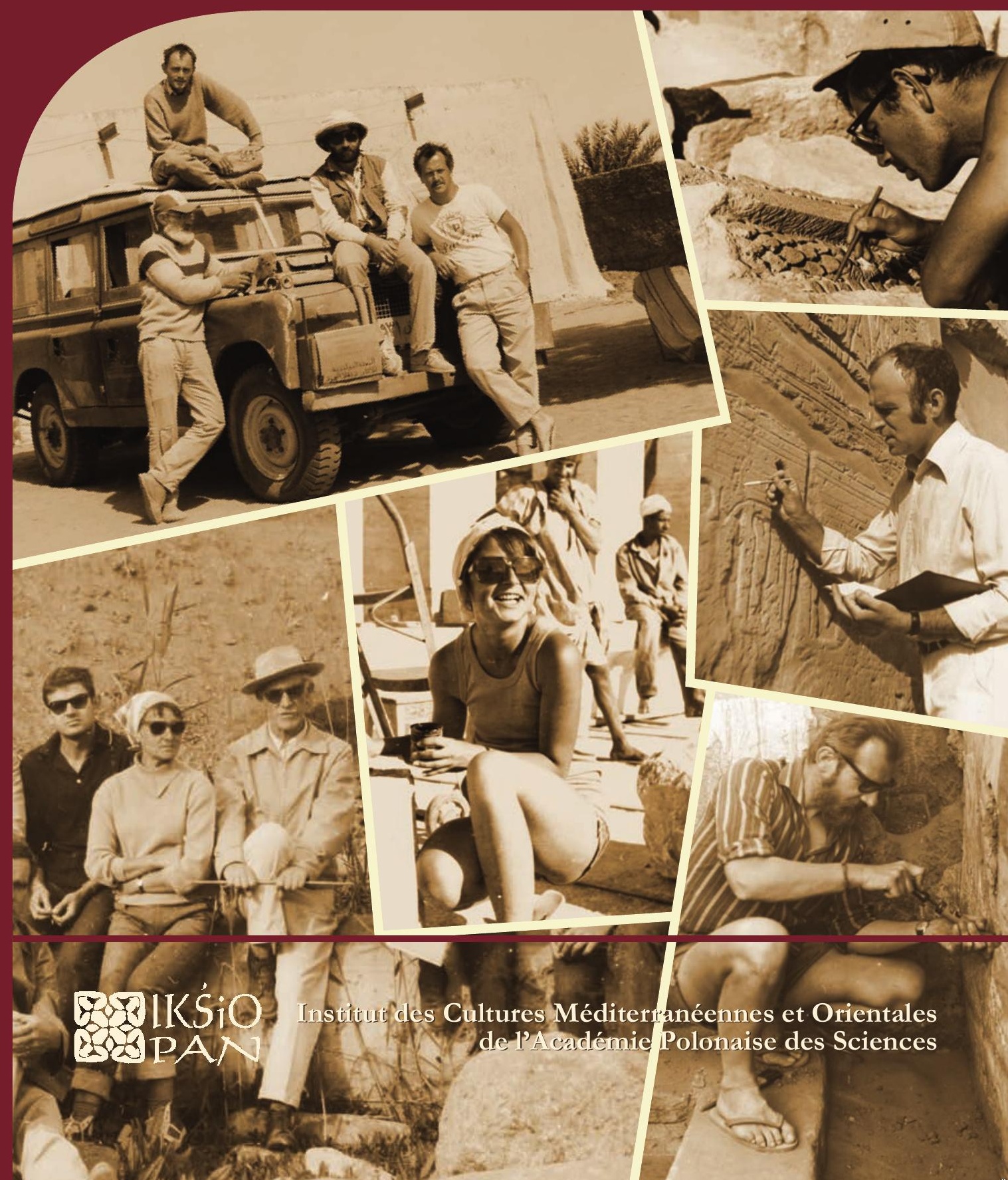




\title{
COMITÉ DE RÉDACTION SCIENTIFIQUE
}

Maciej Makowski - rédacteur en chef

Jadwiga Iwaszczuk - rédacteur et sécretaire de la rédaction

Mariusz Drzewiecki - rédacteur

Maciej G. Witkowski - rédacteur

\section{CONSEIL SCIENTIFIQUE DU JOURNAL}

M. Kobusiewicz (IAE PAS, Warszawa), E. Laskowska-Kusztal (IMOC PAS, Warszawa),

D. Michaelides (University of Cyprus, Nicosia),

J.Ch. Moretti (IRAA-MOM, Université de Lyon 2/CNRS),

D. Raue (Ägyptisches Museum der Universität Leipzig), P. Reynolds (ICREA, Barcelona),

D. Welsby (British Museum, London)

\section{COMITÉ SCIENTIFIQUE DE LECTURE}

J. Holaubek (Institut für Ägyptologie, Wien), S. Ikram (AUC, Cairo),

K. Innemée (Universiteit Leiden), J. McKenzie (Faculty of Oriental Studies, University of Oxford),

N. Strudwick (University of Cambridge), A. Loprieno-Gnirs (Universität Basel),

Ch.E. Loeben (Museen für Kulturgeschichte, Hannover), Y. Tristant (Macquarie University, Sydney),

V.W.J. van Gerven Oei (University of Aberdeen), A. Peignard-Giros (HiSoMA-MOM, Université de Lyon 2/CNRS), J.A. Ostrowski, E. Papuci-Władyka, J. Śliwa (IA JU, Kraków), R. Czerner (WUST, Wrocław), A. Ćwiek (IA AMU, Poznań), M. Wiewióra (IA NCU, Toruń), K. Domżalski

(IAE PAS, Warszawa), K.O. Kuraszkiewicz (DE FOS UW), M. Barwik, P. Bieliński, P. Dyczek, W. Godlewski, D. Ławecka, S. Rzepka, J. Żelazowski, M. Gawlikowski, J. Młynarczyk, A. Niwiński, T. Sarnowski, D. Szeląg, T. Waliszewski (IA UW, Warszawa)

\section{RÉDACTEUR THÉMATIQUE DU VOLUME \\ Barbara Lichocka}

\author{
AIDE RÉDACTION TECHNIQUE \\ Dorota Dobrzyńska, Mariusz Drzewiecki
}

REVUE DES TEXTES ANGLAIS

Jo Harper 
ÉTUDES et TRAVAUX XXX 
INSTYTUT KULTUR ŚRÓDZIEMNOMORSKICH I ORIENTALNYCH POLSKIEJ AKADEMII NAUK

\section{STUDIA i PRACE}

XXX

\section{Ro IKŚSiO \\ ESA PAN}

WARSZAWA

2017 
INSTITUT DES CULTURES MÉDITERRANÉENNES ET ORIENTALES DE L'ACADÉMIE POLONAISE DES SCIENCES

\section{ÉTUDES et TRAVAUX}

XXX

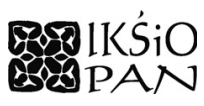

VARSOVIE

2017 
Publication scientifique financée dans le cadre du programme du Ministre de la Science et de l'Éducation Supérieure

« Programme National de Développement de l’Humanistique » pour les années 2016-2021 (projet no 3bH 150099 83)

\title{
HARODOWY PROGRAM ROZWOJU HUMANISTYKI
}

\author{
Copyright (C) \\ Instytut Kultur Śródziemnomorskich i Orientalnych PAN \\ et les Auteurs \\ Warszawa 2017
}

\author{
ISSN 2084-6762 \\ (avant $2011: 0079-3566$ ) \\ e-ISSN 2449-9579 \\ Version première en papier, imprimée en Pologne - 150 copies \\ Version électronique accessible sur \\ http://www.etudesettravaux.iksiopan.pl
}

Édition: Polskie Towarzystwo Historyczne et Wydawnictwo Neriton, Warszawa

Conception générale de couverture : J. Iwaszczuk

Photos de couverture : En haut, à gauche. Vieille Dongola 1991, S. Jakobielski

(debout à gauche), K. Pluskota (debout à droite), B. Żurawski (assis sur le camion)

et P. Wierzbicki (assis sur le camion) (de la collection de B. Żurawski)

En haut, à droite. Palmyre 1964, M. Marciniak au travail (phot. A. Dziewanowski)

Au centre. E. Laskowska-Kusztal au travail (de la collection de E. Laskowska-Kusztal)

En bas, à gauche. Tell Atrib 1962 ; de gauche : T. Biniewski, M. Marciniak, K. Kołodziejczyk,

K. Michałowski, A. Ostrasz, S. Jakobielski et S. Jasiewicz devant eux

(de la collection de IKŚSiO PAN).

En bas, à droite. Vieille Dongola 1976, S. Jakobielski nettoyant le mur (phot. M. Steinborn).

Au centre, à droite, K. Myśliwiec en train des travaux de documentation (de la collection de IKŚiO PAN) 


\section{Table des matières}

BARBARA LICHOCKA

Ergon agathon

Hartwig Altenmüller

$\mathrm{Zu}$ den Feindbildern auf den Zauberstäben des Mittleren Reiches und der Zweiten

Zwischenzeit

Nathalie Beaux

Des $m s w n s w$ de Thoutmosis III à Deir el-Bahari

Briant Bohleke, Nigel Strudwick

A Label for Opening of the Mouth Implements from the Burial of Senneferi (TT99)

and Remarks on the Ritual

Rosa Maria Bonacasa Carra, Nicola Bonacasa

Nuovi dati sugli edifici termali di Sabratha

EDWARD BROVARSKI

A Fragmentary Carrying Chair Scene in Salt Lake City, Utah

Julia Burdajewicz

Wall Painting Decoration from the North-West Church in Hippos-Sussita

of the Decapolis

Mariusz BURDAJEWICZ

From Pagan Temple to Church in Late Antiquity Palestine. A View from

Hippos-Sussita

MAREK ChlodNicki

Early Dynastic Bead Workshops at the Central Kom of Tell el-Farkha.

Patryk ChudziK, Mariusz Caban

Observations on the Architecture of the Tomb of Horhotep in Western Thebes

Krzysztof M. Cialowicz

New Discoveries at Tell el-Farkha and the Beginnings of the Egyptian State.

Amr EL-TiebI

Four Wooden New Kingdom Female Statuettes in the Egyptian Museum, Cairo 


\section{Naguib KanaWATI}

Ritual Marriage Alliances and Consolidation of Power in Middle Egypt during the Middle Kingdom

Adam Łajtar, Jolanta Mlynarczyk

A Faction Acclamation Incised on a Pithos Found Near the North-West Church at Hippos (Sussita)

Adam ŁaJTAR, Grzegorz OchaŁa

Two Private Prayers in Wall Inscriptions in the Faras Cathedral

Adam Łajtar, Anna Poludnikiewicz

Medicinal Vessels from Tell Atrib (Egypt)

JaCeK Michniewicz, Jolanta MlynarczyK

Petrographic Variability of the Fabrics of Wine Jars from Sha'ar-Ha Amakim as a Reflection of Differences in Their Provenance and Chronology

Iwona ModrzewsKa-PianetTI

Les importations d'amphores Dressel 20 en Gaule Cisalpine

Arthur SEgal

Samaria-Sebaste. Portrait of a polis in the Heart of Samaria 409

JOACHIM ŚLIWA

The Motif of a 'Blind Harper' in an Unexpected Place

MONIKA WIĘCH

Searching for the Kitchen in the Early Roman Phase of the 'Hellenistic' House at Nea Paphos (Cyprus)

Abréviations 
THE VOLUME IS PUBLISHED TO CELEBRATE

THE $60^{\text {TH }}$ ANNIVERSARY

OF THE ESTABLISHMENT OF

THE RESEARCH CENTRE FOR MEDITERRANEAN ARCHAEOLOGY POLISH ACADEMY OF SCIENCES

FOUNDED IN 1956

WHOSE MISSION IS CONTINUED BY

THE INSTITUTE OF MEDITERRANEAN AND ORIENTAL CULTURES

OF THE POLISH ACADEMY OF SCIENCES 